\title{
Axonal protection by short-term hyperglycemia with involvement of autophagy in TNF-induced optic nerve degeneration
}

\author{
Kana Sase ${ }^{1}$, Yasushi Kitaoka ${ }^{1,2 *}$, Yasunari Munemasa ${ }^{1}$, Kaori Kojima ${ }^{1}$ \\ and Hitoshi Takagi ${ }^{1}$ \\ ${ }^{1}$ Department of Ophthalmology, St. Marianna University School of Medicine, Kawasaki, Japan, ${ }^{2}$ Department of Molecular \\ Neuroscience, St. Marianna University Graduate School of Medicine, Kawasaki, Japan
}

Previous reports showed that short-term hyperglycemia protects optic nerve axons in a rat experimental hypertensive glaucoma model. In this study, we investigated whether short-term hyperglycemia prevents tumor necrosis factor (TNF)-induced optic nerve degeneration in rats and examined the role of autophagy in this axon change process. In phosphate-buffered saline (PBS)-treated rat eyes, no significant difference in axon number between the normoglycemic (NG) and streptozotocin (STZ)-induced hyperglycemic $(\mathrm{HG})$ groups was seen at 2 weeks. Substantial degenerative changes in the axons were noted 2 weeks after intravitreal injection of TNF in the NG group. However, the HG group showed significant protective effects on axons against TNFinduced optic nerve degeneration compared with the NG group. This protective effect was significantly inhibited by 3-methyladenine (3-MA), an autophagy inhibitor.

OPEN ACCESS

Edited by:

Dirk M. Hermann University Hospital Essen, Germany

Reviewed by: Ulkan Kilic, Istanbul Medipol University, Turkey Arne Herring, University Hospital Essen, Germany

${ }^{*}$ Correspondence: Yasushi Kitaoka kitaoka@marianna-u.ac.jp

Received: 15 August 2015 Accepted: 09 October 2015 Published: 28 October 2015

Citation: Sase K, Kitaoka Y, Munemasa Y, Kojima $K$ and Takagi H (2015) Axonal protection by short-term hyperglycemia with involvement of autophagy in TNF-induced optic nerve degeneration.

Front. Cell. Neurosci. 9:425. doi: 10.3389/fncel.2015.00425
Immunoblot analysis showed that the LC3-II level in the optic nerve was increased in the HG group compared with the NG group. Increased p62 protein levels in the optic nerve after TNF injection was observed in the NG group, and this increase was inhibited in the HG group. Electron microscopy showed that autophagosomes were increased in optic nerve axons in the HG group. Immunohistochemical study showed that LC3 was colocalized with nerve fibers in the retina and optic nerve in both the NG and HG groups. Short-term hyperglycemia protects axons against TNF-induced optic nerve degeneration. This axonal-protective effect may be associated with autophagy machinery.

Keywords: hyperglycemia, autophagy, LC3, p62, tumor necrosis factor, optic nerve

\section{INTRODUCTION}

Glaucoma is a cause of visual field defect due to loss of nerve fibers and thinning of the neuronal rim of the optic nerve head. The risk factors for glaucoma were defined in epidemiological studies (Leske et al., 1994, 2008; Sommer and Tielsch, 1996; Francis et al., 2008; Topouzis et al., 2011). Ocular hypertension, family history, older age, and thinner central corneal thickness

\footnotetext{
Abbreviations: NG, normoglycemic; HG, hyperglycemic; STZ, streptozotocin; LC3, microtubule-associated protein light chain 3; POAG, primary open-angle glaucoma; RGC, retinal ganglion cell; TNF, tumor necrosis factor; PBS, phosphate-buffered saline; 3-MA, 3-methyladenine; DAPI, 4',6-diamidino-2-phenylindole; DMSO, dimethyl sulfoxide; TBS, Tris-buffered saline; PSS, physiological saline solution.
} 
are risk factors for primary open-angle glaucoma (POAG; Leske et al., 1994, 2008; Sommer and Tielsch, 1996; Coleman and Miglior, 2008; Francis et al., 2008; Topouzis et al., 2011). It is believed that diabetes mellitus (DM) also influences the pathogenesis and progression of glaucoma (Klein et al., 1994; Leske et al., 1995; Tielsch et al., 1995; Mitchell et al., 1997; Chopra et al., 2008), although the relationship between DM and glaucoma remains controversial. Some recent meta-analyses have shown that DM is risk factor for glaucoma (Zhou et al., 2014; Zhao et al., 2015). It was determined that the pooled relative risk of the association between DM and POAG based on the risk estimate of six cohort studies was 1.40 (Zhou et al., 2014). In contrast, some studies found that glaucoma development is inhibited by DM (Gordon et al., 2002; Akkaya et al., 2014). The Ocular Hypertension Treatment Study showed that among the 191 participants who reported a history of DM at baseline, 6 (3.1\%) developed POAG compared with $119(8.3 \%)$ of 1427 participants who did not report a history of DM (Gordon et al., 2002). The univariate hazard ratio for DM was 0.40 and multivariate hazard ratio was 0.37 (Gordon et al., 2002). Moreover, a recent study using Heidelberg retina tomography III has found that rim area and rim volume were significantly higher in POAG patients with DM compared with those without DM and suggested the protective effects of DM against glaucomatous optic nerve damage (Akkaya et al., 2014). Furthermore, a recent double-blind randomized study has shown that $50 \%$ glucose eye drops significantly improved the mean contrast sensitivity compared with $0.9 \%$ saline in individuals with POAG (Casson et al., 2014). In the animal models, short-term hyperglycemia attenuated axonal degeneration and retinal ganglion cell (RGC) death after experimentally induced ocular hypertension (Ebneter et al., 2011). Although the results of that study suggested that the inhibition of microglial activation is involved in the protective effect of hyperglycemia (Ebneter et al., 2011), its detailed mechanism remains to be elucidated.

Autophagy, or cellular self-digestion, is a cellular pathway involved in protein and organelle degradation which is associated with the pathophysiology of several human diseases (Mizushima et al., 2008). In particular, impaired autophagy has been associated with chronic central nervous system (CNS) diseases (Margulis and Finkbeiner, 2014; Menzies et al., 2015). Recent studies have shown that autophagy is activated by short-term hyperglycemia in Purkinje cells (Yang et al., 2014) and the gastrocnemius (Lv et al., 2014) in rats, and in astrocytes in the brain in mice (Zhang et al., 2010). We have recently demonstrated that the activation of autophagy leads to axonal protection in the hypertensive experimental glaucoma model (Kitaoka et al., 2013) and in the tumor necrosis factor (TNF)induced optic nerve degeneration model (Kojima et al., 2014). TNF is involved in certain types of glaucoma (Tezel and Wax, 2000; Yan et al., 2000; Yuan and Neufeld, 2000; Tezel et al., 2001; Nakazawa et al., 2006; Sawada et al., 2010), and the TNF injection model may be useful in understanding the mechanism of axonal degeneration of RGCs (Kitaoka et al., 2006; Munemasa and Kitaoka, 2013). Therefore, in the present study, we investigated whether short-term hyperglycemia prevents TNF-induced optic nerve degeneration and examined the role of autophagy in this axon change process.

\section{MATERIALS AND METHODS}

\section{Animals}

Experiments were performed on 8-week-old male Wistar rats. All studies were conducted according to the Association for Research in Vision and Ophthalmology (ARVO) statement on the Use of Animals in Ophthalmic and Vision Research and were approved by the Ethics Committee of the Institute of Experimental Animals of St. Marianna University Graduate School of Medicine. The animals were housed in controled conditions, with temperature at $23 \pm 1^{\circ} \mathrm{C}$, humidity at $55 \pm 5 \%$, and light from 06:00-18:00.

\section{Normoglycemic (NG) and Hyperglycemic (HG) Models}

The rats were divided into two groups. One group received an intraperitoneal injection of physiological saline solution (PSS) to serve as the normoglycemic (NG) group and the other received an intraperitoneal injection of $60 \mathrm{mg} / \mathrm{kg}$ streptozotocin (STZ; Wako, Osaka, Japan) to serve as the HG group. Plasma glucose levels were measured 4 days after intraperitoneal injection using One Touch Ultra View (Johnson \& Johnson, Tokyo, Japan). Rats with a plasma glucose level of less than $250 \mathrm{mg} / \mathrm{dl}$ in the HG group were excluded from analysis. Plasma glucose levels were also measured 1 and 2 weeks after intravitreal injection.

\section{Administration of TNF}

Both the NG and HG groups received an intravitreal injection of TNF 4 days after intraperitoneal injection of PSS or STZ. Intravitreal injection of TNF was performed as described previously (Kitaoka et al., 2006, 2009). Briefly, rats were anesthetized with an intramuscular injection of a mixture of ketamine-xylazine (10 and $4 \mathrm{mg} / \mathrm{kg}$, respectively). A single $2-\mu \mathrm{l}$ injection of $10 \mathrm{ng}$ TNF in $0.01 \mathrm{M}$ PBS, pH 7.40, was administered intravitreally to the right eye of an animal under a microscope to avoid lens injury. Phosphate-buffered saline (PBS) alone was administered to the contralateral left eye as a control. In the HG group, 60 mM 3-methyladenine (3-MA; Sigma-Aldrich, St. Louis, MO, USA), an autophagy inhibitor, in dimethyl sulfoxide (DSMO) was administered simultaneously with TNF into the right eyes. The rats were euthanized 1 or 2 weeks after the intravitreal injections with an intraperitoneal overdose of sodium pentobarbital, followed by enucleation of the eyes.

\section{Immunoblot Analysis}

Eighty-four rats were used for immunoblot analysis as described previously (Kitaoka et al., 2011). Briefly, 1 week after intravitreal TNF and PBS injection, optic nerves $(4 \mathrm{~mm}$ in length starting from immediately behind the globe) were collected, homogenized, and then centrifuged at $15,000 \times g$ for $15 \mathrm{~min}$ at $4^{\circ} \mathrm{C}$. Two optic nerve specimens were pooled into one sample. Protein concentrations were determined using the BioRad Protein Assay kit (Bio-Rad, Hercules, CA, USA). Protein samples $(2.5 \mu \mathrm{g}$ per lane) were subjected to SDS-PAGE on gels 
(Bio-Rad) and transferred to PVDF membranes (ImmobilonP, Millipore, Billerica, MA, USA). Membranes were blocked with Tris-buffered saline (TBS) $-0.1 \%$ Tween-20 containing 5\% skim milk. Membranes were first reacted with anti-microtubuleassociated protein light chain 3 (LC3) antibody (1:200; Medical \& Biological Laboratories Co., Nagoya, Japan), anti-p62 antibody (1:200; Medical and Biological Laboratories Co.), or anti- $\beta$ actin antibody (1:500; Sigma-Aldrich) in TBS containing 5\% skim milk. Membranes were then sequentially exposed to peroxidase-labeled anti-rabbit IgG antibody (Cappel, Solon, $\mathrm{OH}$, USA) or peroxidase-labeled anti-mouse IgG antibody (Cappel) diluted 1:5000 in Tween-20 in TBS. Western blots were visualized with an ECL detection system (Amersham ECL Prime Western Blotting Detection Reagents, GE Healthcare, Buckinghamshire, UK).

\section{Immunohistochemistry}

Seven rats in total were used for immunohistochemical study. One week after PBS or TNF injection, optic nerve samples from the NG (3 rats) and HG (4 rats) groups were fixed by immersion in 10\% neutral-buffered formalin for $24 \mathrm{~h}$, dehydrated, embedded in paraffin, and sectioned (4 $\mu \mathrm{m}$ thick). Deparaffinized sections were incubated with $1 \%$ bovine serum and then reacted with primary antibodies against LC3 (1:200; Medical \& Biological Laboratories Co.), or neurofilament-L (a marker of neurons; 1:100; DAKO Corporation, Carpinteria, CA, USA) diluted in $1 \%$ bovine serum overnight at $4^{\circ} \mathrm{C}$. Sections were then exposed to the following secondary antibodies: FITClabeled anti-rabbit antibody (1:100; Cappel, MP Biomedicals, LLC) or rhodamine-labeled anti-mouse antibody (1:100; Cappel, MP Biomedicals, LLC). The samples were counterstained with 4',6-diamidino-2-phenylindole (DAPI; Vesctashield with DAPI, Vector Laboratories, Inc., Burlingame, CA, USA). Negative controls were performed by replacing the primary antibody with PBS or serum.

\section{Axon Counting}

Morphometric analysis of each optic nerve was performed as described previously with samples from 17 rats (Kitaoka et al., 2009, 2011; Kojima et al., 2014). Eyes were obtained from the animals 2 weeks after intravitreal injection. Four-millimeter segments of the optic nerves were obtained starting from $1 \mathrm{~mm}$ behind the globe. These segments of optic nerve were fixed by immersion in Karnovsky's solution for $24 \mathrm{~h}$ at $4^{\circ} \mathrm{C}$, processed, and embedded in acrylic resin. Cross sections ( $1 \mu \mathrm{m}$ thick) were cut beginning $1 \mathrm{~mm}$ from the globe and stained with a solution of $1 \%$ paraphenylen-diamine (Sigma-Aldrich) in absolute methanol. For each section, images at the center and at each quadrant of the periphery (approximately $141.4 \mu \mathrm{m}$ from the center) were acquired with a light microscope (BX51; Olympus, Tokyo, Japan) with a $100 \times$ coupled digital camera (MP5Mc/OL; Olympus) and associated QCapture Pro software (version 5.1, QImaging, Surrey, Canada). The acquired images were quantified using Aphelion image-processing software (version 3.2, ADISC SA and AAI, Inc., Herouville Saint Clair, France). The number of axons was determined in five distinct areas of $1446.5 \mu \mathrm{m}^{2}$ each (each quadrant of the periphery in addition to the center; total area of $7232.3 \mu \mathrm{m}^{2}$ per eye) from each eye. The number of axons per eye was averaged and expressed as the number per square millimeter. A minimum of four eyes per experimental condition was used for analysis.

\section{Electron Microscopy}

Eyes were obtained from animals 2 weeks after intravitreal injection. Four-millimeter segments of the optic nerves were obtained starting from $1 \mathrm{~mm}$ behind the globe. These segments of optic nerve were fixed by immersion in Karnovsky's solution for $24 \mathrm{~h}$ at $4^{\circ} \mathrm{C}$, processed, and embedded in acrylic resin. Ultrathin sections (approximately $100 \mathrm{~nm}$ thick) were prepared using an LKB Ultrotome V (LKB-Produkter AB, Bromma, Sweden), contrasted with saturated aqueous uranyl acetate and Sato's lead solution, and viewed with a JEOL 1200EX transmission electron microscope (JEOL, Tokyo, Japan) at $80 \mathrm{kV}$. The number of autophagosomes was determined in 10 distinct areas of $157.3 \mu \mathrm{m}^{2}$ each (total area of $1573 \mu \mathrm{m}^{2}$ per eye) from each eye. Three eyes per experimental condition were used for analysis.

\section{Statistical Analysis}

For plasma glucose, data are presented as mean \pm S.D. Other data are presented as mean \pm S.E.M. Differences among groups were analyzed using Mann-Whitney's method. A probability value of $<0.05$ was considered to represent a statistically significant difference.

\section{RESULTS}

\section{Effects of STZ on Plasma Glucose Levels}

There was no significant difference in baseline plasma glucose levels (immediately before intraperitoneal injection, day 0 ) between the PSS-treated $(122.6 \pm 6 \mathrm{mg} / \mathrm{dl}, n=5)$ and STZ-treated groups $(121 \pm 6.6 \mathrm{mg} / \mathrm{dl}, n=6)$. The plasma glucose levels of STZ-treated HG rats were significantly increased from 4-18 days after intraperitoneal injection of STZ compared with those of PSS-treated NG rats $(436.6 \pm 59.2 \mathrm{mg} / \mathrm{dl}$ and $137 \pm 7.5 \mathrm{mg} / \mathrm{dl}$, STZ and PSS groups, respectively, at 4 days, i.e., immediately before intravitreal injection, $n=5-6$ per group, $p<0.01 ; 477.5$ $\pm 42.1 \mathrm{mg} / \mathrm{dl}$ and $172.4 \pm 27.6 \mathrm{mg} / \mathrm{dl}$, STZ and PSS groups, respectively, at 11 days, i.e., 1 week after intravitreal injection, $n=5-6$ per group, $p<0.01 ; 522.1 \pm 60.1 \mathrm{mg} / \mathrm{dl}$ and $136.6 \pm 21.9$ $\mathrm{mg} / \mathrm{dl}$, STZ and PSS groups, respectively, at 18 days, i.e., 2 weeks after intravitreal injection, $n=5-6$ per group, $p<0.01$ ).

\section{Effects of Hyperglycemia and 3-MA on TNF-Induced Axonal Degeneration}

In PBS-injected eyes, there was no significant difference in axon number between the $\mathrm{HG}$ and $\mathrm{NG}$ groups (Figures $\mathbf{1 A}, \mathbf{C}, \mathbf{F}$ ). Compared with PBS-treated eyes, we confirmed substantial degenerative changes in the axons in the NG group 2 weeks after TNF injection (Figure 1B), which is consistent with the findings of our previous studies (Kitaoka et al., 2006, 2009). The axonal loss after TNF injection was 35\% compared with PBS-injected eyes (Figure 1F). However, HG induction exerted 

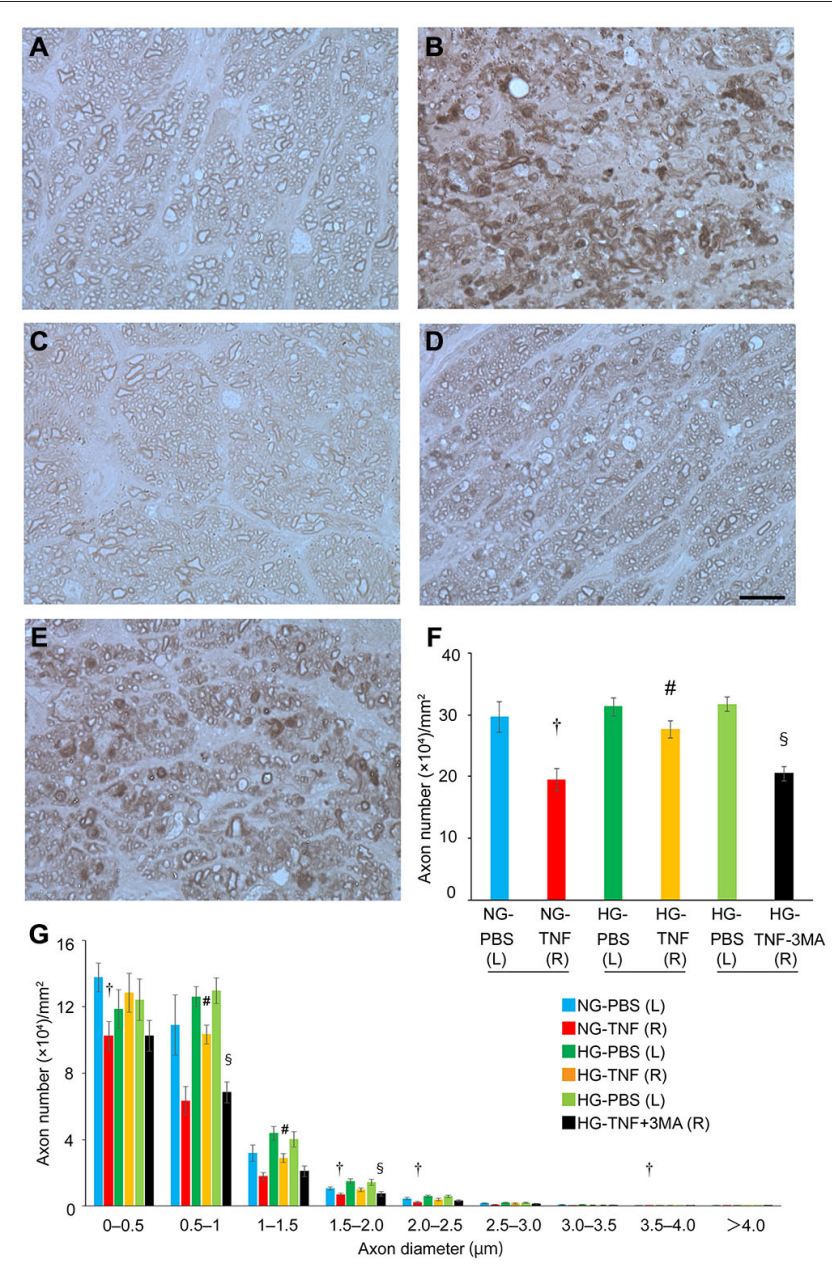

FIGURE 1 | Short-term hyperglycemia prevented axon loss in TNF-induced optic nerve degeneration. Light microscopic findings 2 weeks after (A) PBS injection with NG, (B) 10-ng TNF injection with NG, (C) PBS injection with HG, (D) 10-ng TNF injection with HG, or (E) 10-ng TNF injection \pm MA with $\mathrm{HG}$. Scale bar $=10 \mu \mathrm{m}(\mathbf{A}-\mathbf{E})$. Effect of hyperglycemia, TNF, and 3-MA on axon numbers (F) and distribution of axon diameters (G) in the optic nerves. Each column represents mean \pm S.E.M.; $n=4-7$ eyes per group. ${ }^{\dagger} p<0.05$ compared with PBS injection with NG; ${ }^{\#} p<0.05$ compared with TNF injection with NG; ${ }^{\S} p<0.05$ compared with TNF injection with $\mathrm{HG}$. $(L)$, left eyes; $(R)$, right eyes; paired $(L)$ and $(R)$ indicate the same animal.

a significant protective effect against TNF-induced axonal loss (Figures 1D,F). The HG group showed $84.5 \%$ axonal protection compared with the NG group after TNF injection. Similar trends were observed in axon diameter profiles (Figure 1G), although no specific findings were dependent on axon diameter.

Next, we determined the involvement of autophagy in this protective process. Since previous reports showed that autophagy is activated by short-term hyperglycemia (Zhang et al., 2010; Lv et al., 2014; Yang et al., 2014), we examined whether an autophagy inhibitor would affect this axonal protection. As shown in Figure 1E, the protective effect was significantly inhibited by 3-MA, an autophagy inhibitor (Figure 1F). This was also confirmed in axon diameter profiles (Figure 1G).

\section{Effects of Hyperglycemia, TNF, and 3-MA on LC3-II and p62 Levels in Optic Nerves}

To investigate the effects of hyperglycemia on autophagic status, we examined the changes in LC3-II, an autophagic marker, and $\mathrm{p} 62$, a multifunctional protein that interacts with a central component of the autophagy machinery, in the optic nerve. We previously found that axonal loss started 1 week after TNF injection (Kitaoka et al., 2006), and the molecular events before axon loss becomes obvious are important for clarifying the mechanism of axonal degeneration. In PBS- and TNFinjected eyes, there were marked increases in LC3-II protein levels in the optic nerve in HG groups compared with NG groups (Figure 2A). The increase in LC3-II protein levels in HG group was significantly inhibited by 3-MA.

In the NG groups, there was a substantial increase in p62 protein levels in optic nerve samples 1 week after TNF injection (Figure 2B), which is consistent with our previous report in Kojima et al. (2014). In PBS-treated eyes, there was no significant difference in p62 protein levels in the optic nerve between the NG and HG groups (Figure 2B). However, the TNF-induced upregulation of p62 protein levels in the optic nerve in the NG

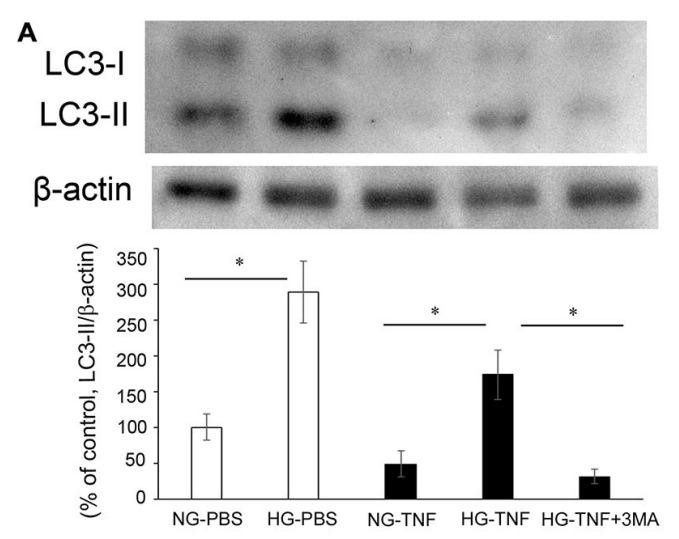

B
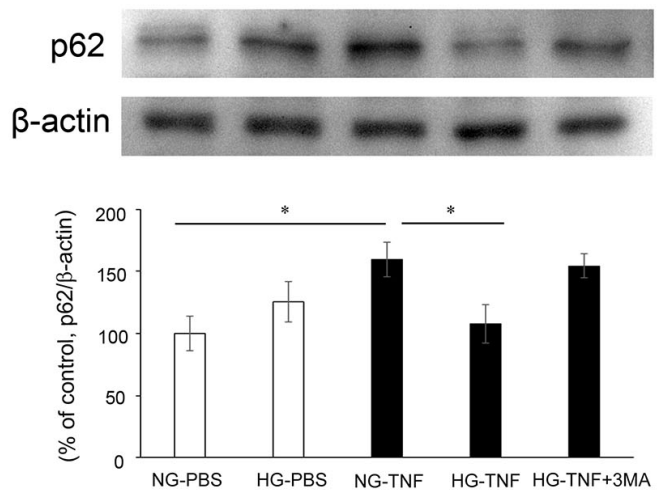

FIGURE 2 | LC-3 II and p62 protein levels in optic nerves. Immunoblot data are normalized to $\beta$-actin levels in the same sample. All data are expressed as a percentage of control. Each column represents mean \pm S.E.M. (A) Immunoblotting for LC-3 II 1 week after intravitreal injection. $n=5-7$ (10-14 eyes) per group. * $p<0.05$. (B) Immunoblotting for p62 1 week after intravitreal injection. $n=6-9\left(12-18\right.$ eyes) per group. ${ }^{*} p<0.05$. 
group was significantly inhibited in the HG group (Figure 2B). This inhibitory effect of $\mathrm{HG}$ induction was not observed in combination with 3-MA treatment (Figure 2B).

\section{Effects of Hyperglycemia and TNF on Electron Microscopy Findings}

In the NG-PBS group, normal structures of microtubules, neurofilaments, and myelin were observed, but autophagosomes were few (Figures 3A,B). In the HG-PBS group, some autophagosomes were observed inside axons in $\varepsilon$ the optic nerve and the structures of microtubules, neurofilaments, and myelin were normal (Figures 3C,D). Consistent with our light microscopy findings showing that degenerative changes were apparent, degenerative changes such as neurofilament accumulation and myelin disorganization were observed in the NG-TNF group (Figures 3E,F). However, these degenerative changes were inhibited and well-preserved myelin and microtubule structures were observed in the HG-TNF group (Figures 3G,H). In addition, some autophagosomes were also observed in the HG-TNF group (Figures $\mathbf{3 G}, \mathbf{H}$ ). We counted autophagsome numbers inside axons of the optic nerve. In both PBS- and TNF-injected eyes, there were significant increases in autophagosome numbers in the optic nerves in HG groups compared with NG groups (Figure 3I). These findings are consistent with the substantial increases in LC3-II protein levels seen in the optic nerve in HG groups.

\section{LC3 in Nerve Fibers in the Retina and Optic Nerve}

As previously reported in Kim et al. (2008), we observed LC3 immunoreactivity in RGCs in the retina (Figures 4A-E). Doublelabeling immunohistochemical studies showed substantial colocalization of LC3 and neurofilaments in the retina in NG and $\mathrm{HG}$ rats (Figures $\mathbf{4 A - E}$ ), suggesting that LC3 is present not only in RGC bodies but also in their axons. Notably, LC3-immunopositive dots were observed inside neurofilamentpositive fibers (Figure 4C). In addition, LC3 immunoreactivity appeared to increase in the optic nerves in the HG-TNF group compared with that in the NG-TNF group (Figures 5A,B). Substantial colocalization of LC3 and neurofilaments was also found in the optic nerves (Figures 5A,B).

\section{DISCUSSION}

The present study demonstrated that short-term (2-week) hyperglycemia does not cause axonal degeneration but instead exerts axonal protection against TNF-induced optic nerve degeneration as shown by morphometric analysis of light microscopic as well as by electron microscopic study results. This finding is consistent with previous results showing that short-term hyperglycemia protects retinal neurons including RGCs against ischemic injury (Casson et al., 2004) and protects RGCs and optic nerve axons against ocular hypertension injury (Ebneter et al., 2011). It is also interesting to note that a recent study has demonstrated that glucose rescued cultured rat retinal cells from rotenone-induced toxicity via both the

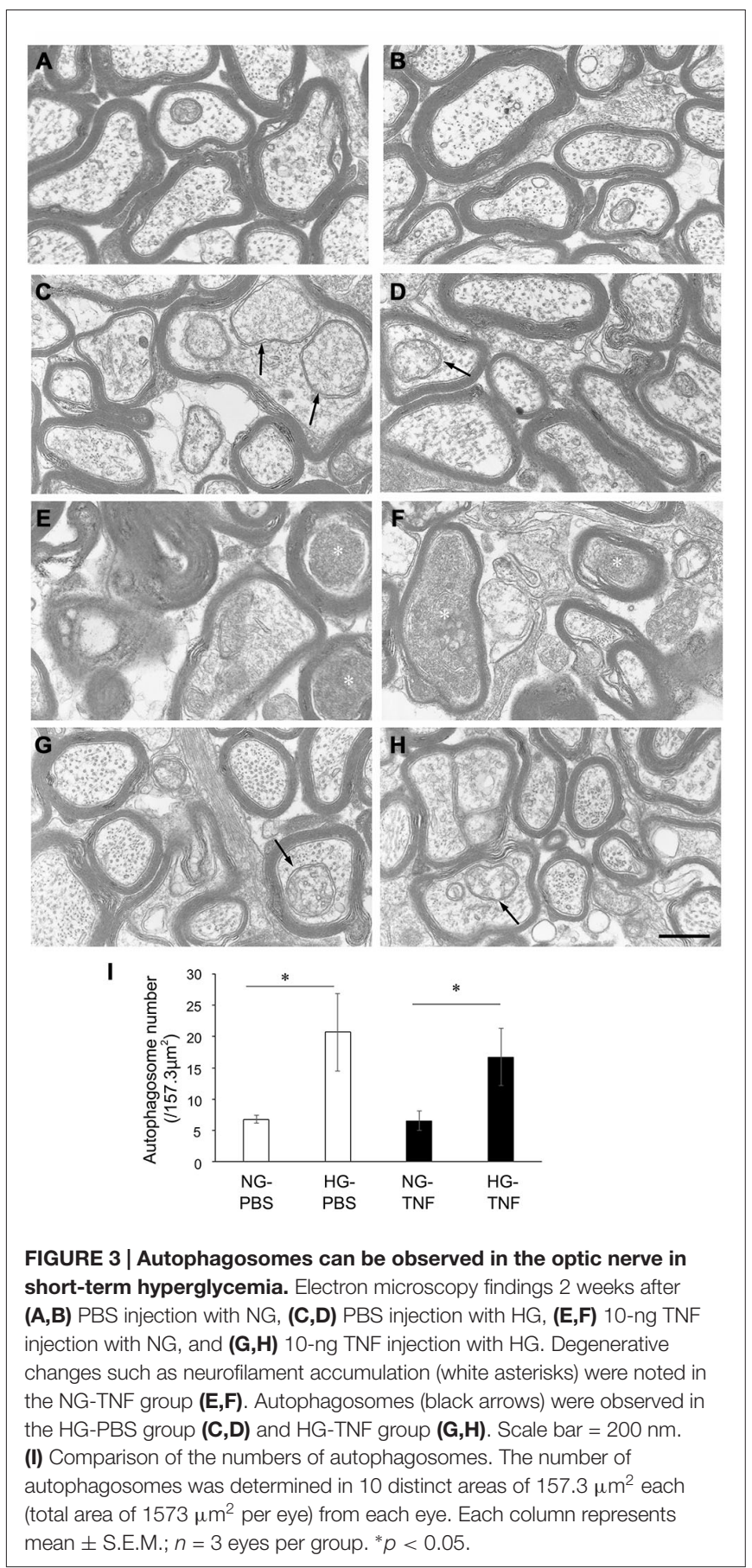

pentose phosphate pathway and the glycolytic pathway, with the maintenance of ATP levels and reduced reactive oxygen species production (Han et al., 2013). On the other hand, we previously found that astrocytes in the optic nerve were activated after TNF injection (Kojima et al., 2012). Therefore, one hypothesis is that TNF enhances glucose utilization by astrocytes and can perturb their energy metabolism (Yu et al., 1995), thereby impairing the ability to provide adequate energy for axons.

The roles of autophagy using rapamycin, an autophagy inducer, and 3-MA, an autophagy inhibitor, on optic nerve 


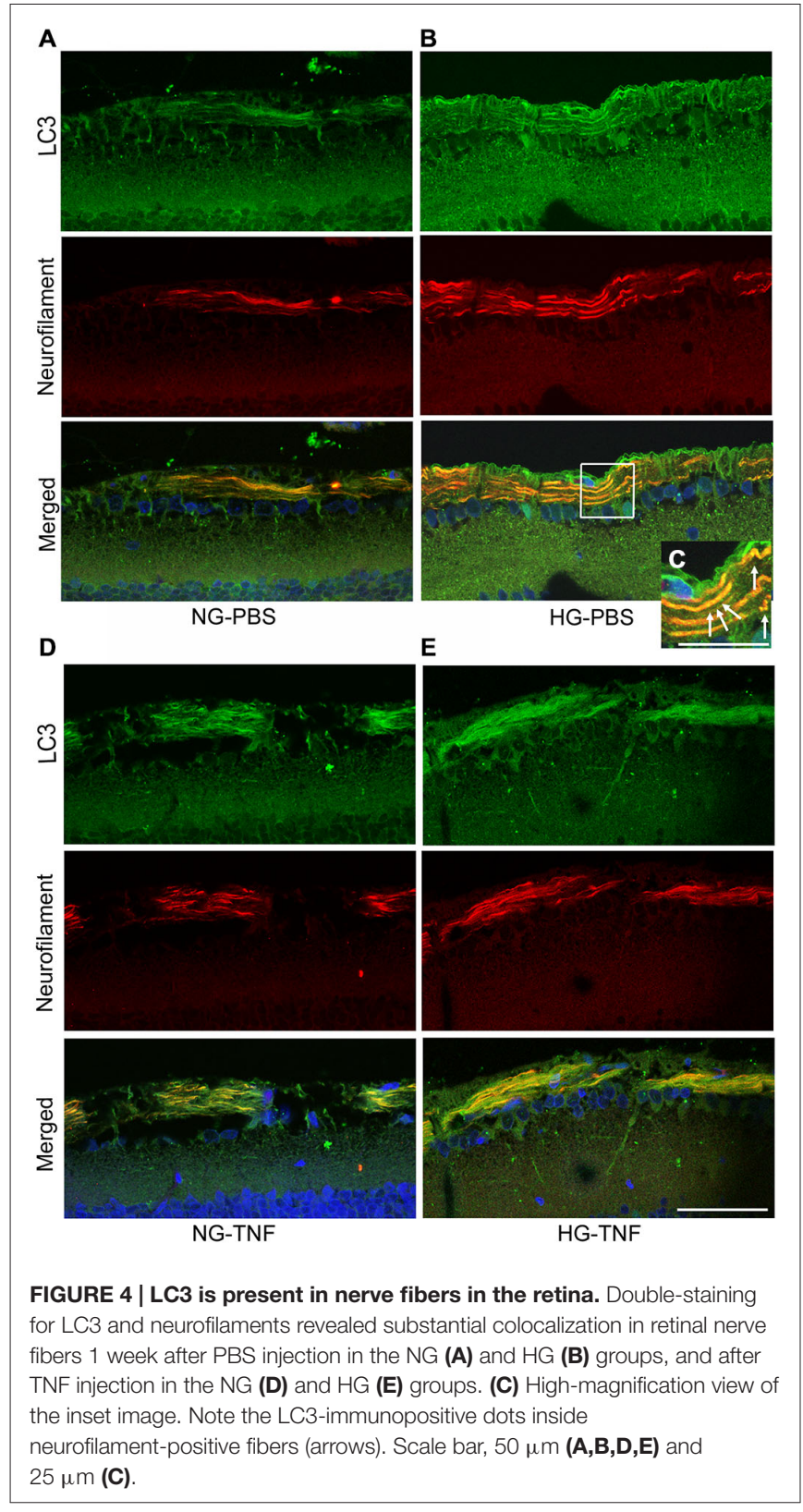

axons and RGCs in different injuries remain controversial (Knöferle et al., 2010; Park et al., 2012; Rodríguez-Muela et al., 2012; Deng et al., 2013). However, our present study showed that the protective effect of short-term hyperglycemia was significantly inhibited by 3-MA, suggesting that autophagic machinery mediates this protective effect. Moreover, our recent findings that rapamycin exerts axonal protection against TNFinduced optic nerve degeneration (Kojima et al., 2014) also support the current findings. In addition, it is noteworthy that high glucose-induced autophagy protects bone marrow-derived mesenchymal stem cells from apoptosis (Chang et al., 2015).

The amount of LC3-II correlates closely with autophagosome number (Mizushima et al., 2010). Our immunoblot analysis showed substantial increases in LC3-II protein levels in the optic nerve in $\mathrm{HG}$ groups compared with NG groups. Consistent

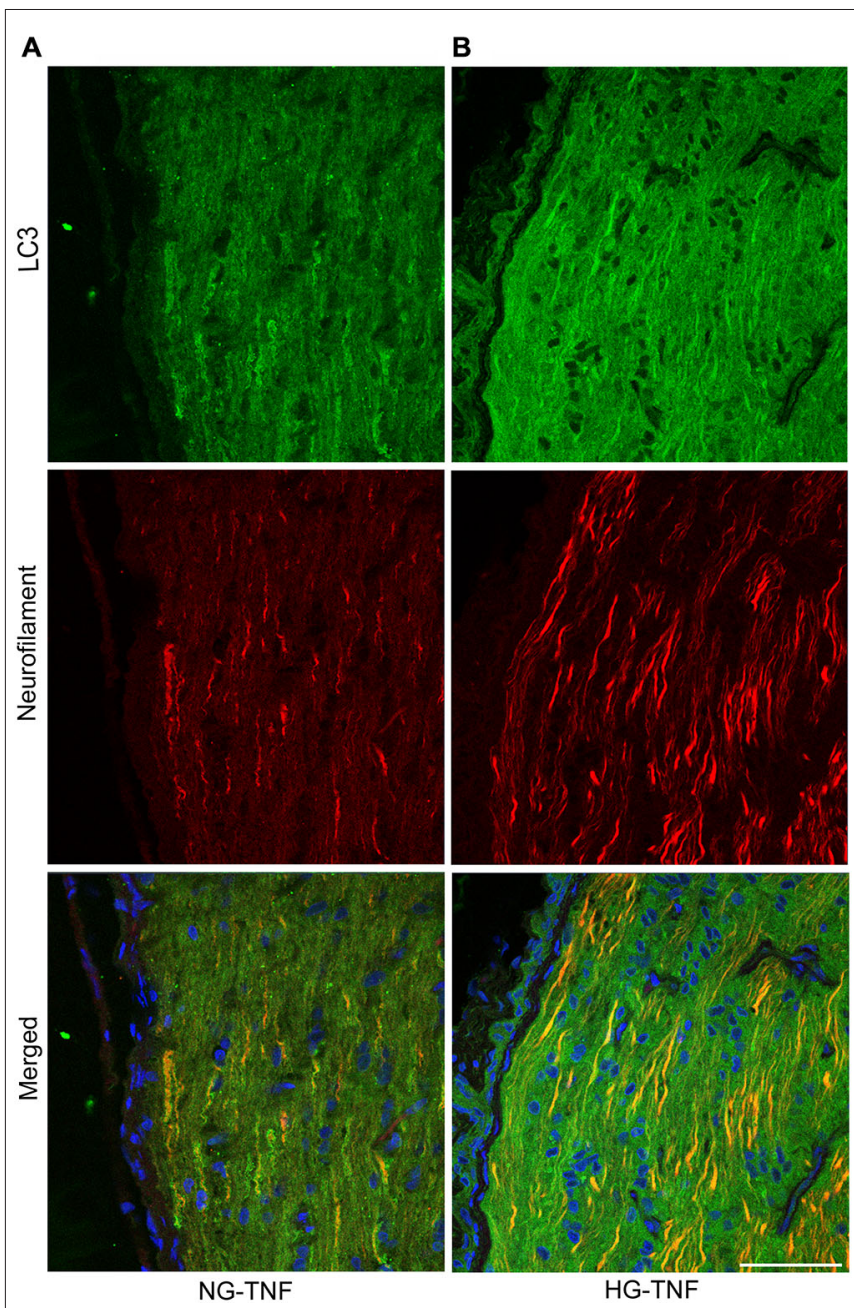

FIGURE 5 | LC3 is present in the optic nerve. Double-staining for LC3 and neurofilaments revealed substantial colocalization in the optic nerve 1 week after TNF injection in the NG (A) and HG (B) groups. Scale bar, $50 \mu \mathrm{m}$.

with this, the present electron microscopic study showed that noticeable autophagosomes were observed in both the HG-PBS and HG-TNF groups and quantification confirmed that their number increased. STZ is an antibiotic that can cause pancreatic $\beta$-cell destruction ( $\mathrm{Wu}$ and Huan, 2008), thereby leading to low levels of plasma insulin. A previous report showed that under HG conditions induced by glucose infusion, autophagy was inhibited in rat skeletal muscle, whereas under STZ-induced HG conditions, autophagy was enhanced (Lv et al., 2014). This difference in autophagic status suggests that insulin may regulate autophagy machinery (Lv et al., 2014). During hyperglycemia, the administration of insulin reduced LC3-II levels in type 2 diabetes patient muscle (Kruse et al., 2015), implying that low levels of insulin may lead to increased LC3-II levels. Taken together, the results suggest that the increases in LC3-II protein levels in the optic nerve may be associated with autophagy activation in STZ-induced HG rats that have low levels of insulin.

We observed LC3-immunopositive dots inside neurofilament-positive fibers near RGC bodies and in the 
optic nerve. Since LC3 is known to occur on autophagosomes and widely used as a marker of autophagosomes (Kabeya et al., 2000; Mizushima et al., 2010), the presence of LC3 in nerve fibers is consistent with our electron microscopy findings indicating the presence of autophagosomes inside axons. It is particularly important to note that autophagosome biogenesis occurs in distal neurites and then moves mainly retrogradely to cell soma in dorsal root ganglia neurons (Maday et al., 2012). Although it is not clear whether this is the case for optic nerve axons and RGC soma, it is possible that increased LC3 protein levels in the optic nerve resulted in abundant LC3-immunopositive dots in all neurofilament-positive fibers in the HG group.

A previous report showed that hyperglycemia does not affect p62 protein levels in human muscle (Kruse et al., 2015). However, in the present study, the short-term hyperglycemia significantly inhibited TNF-induced upregulation of p62 protein levels in the optic nerve. Consistent with this finding, a previous study demonstrated that the p62 protein level in brain tissue was significantly decreased in STZ-induced hyperglycemia compared with normoglycemia in a cerebral ischemic mouse model (Wei et al., 2013). Since decreased p62 levels indicate enhanced autophagic flux (Bjørkøy et al., 2009), one possibility is that STZ-induced hyperglycemia may lead to increased autophagic flux in the optic nerve. Because we observed a difference in the p62 level between the NG and HG groups only in TNF-injected eyes, hyperglycemia may improve autophagic flux in TNF-induced optic nerve degeneration, although this may not occur in PBS-injected eyes. It is proposed that enhanced autophagic flux leads to increasing clearance of unnecessary

\section{REFERENCES}

Akkaya, S., Can, E., and Oztürk, F. (2014). Comparison of optic nerve head topographic parameters in patients with primary open-angle glaucoma with and without diabetes mellitus. J. Glaucoma doi: 10.1097/ijg.0000000000000096 [Epub ahead of print].

Bjørkøy, G., Lamark, T., Pankiv, S., Øvervatn, A., Brech, A., and Johansen, T. (2009). Monitoring autophagic degradation of p62/SQSTM1. Methods Enzymol. 452, 181-197. doi: 10.1016/s0076-6879(08) 03612-4

Casson, R. J., Chidlow, G., Wood, J. P., and Osborne, N. N. (2004). The effect of hyperglycemia on experimental retinal ischemia. Arch. Ophthalmol. 122, 361-366. doi: 10.1001/archopht.122.3.361

Casson, R. J., Han, G., Ebneter, A., Chidlow, G., Glihotra, J., Newland, H., et al. (2014). Glucose-induced temporary visual recovery in primary open-angle glaucoma: a double-blind, randomized study. Ophthalmology 121, 1203-1211. doi: 10.1016/j.ophtha.2013.12.011

Chang, T. C., Hsu, M. F., and Wu, K. K. (2015). High glucose induces bone marrow-derived mesenchymal stem cell senescence by upregulating autophagy. PLoS One 10:e0126537. doi: 10.1371/journal.pone. 0126537

Chopra, V., Varma, R., Francis, B. A., Wu, J., Torres, M., and Azen, S. P. (2008). Type 2 diabetes mellitus and the risk of open-angle glaucoma the los angeles latino eye study. Ophthalmology 115, 227-332. doi: 10.1016/j.ophtha.2007. 04.049

Coleman, A. L., and Miglior, S. (2008). Risk factors for glaucoma onset and progression. Surv. Ophthalmol. 53, S3-S10. doi: 10.1016/j.survophthal.2008. 08.006

Deng, S., Wang, M., Yan, Z., Tian, Z., Chen, H., Yang, X., et al. (2013). Autophagy in retinal ganglion cells in a rhesus monkey chronic hypertensive glaucoma model. PLoS One 8:e77100. doi: 10.1371/journal.pone.0077100 proteins and therefore may be beneficial for neuroprotective interventions in optic neuropathy (Munemasa and Kitaoka, 2015). We only observed axonal protection over the short term and expect that long-term hyperglycemia will lead to neurodegeneration, as shown in other neuronal cells that exhibited autophagic activity impairment and degeneration in the Purkinje neurons of 24-week STZ-HG rats (Yang et al., 2014).

In conclusion, our present results suggest that short-term hyperglycemia protects axons in TNF-induced optic nerve degeneration and that this axonal-protective effect may be associated with autophagy machinery.

\section{AUTHOR CONTRIBUTIONS}

KS, YK, YM and KK performed experiments; KS, YK and HT designed the research protocol and analyzed data; and KS, YK and KK wrote and YM and HT revised the article. All authors have read and approved the final manuscript.

\section{ACKNOWLEDGMENTS}

This work was supported by Grants-in-Aid No. 15K10908 (YK) and No. 25462739 (YM) from the Ministry of Education, Culture, Sports, Science and Technology of Japan; the Suzuken Memorial Foundation (YK); and Charitable Trust Fund for Ophthalmic Research in Commemoration of Santen Pharmaceutical's Founder (YK). We thank Ms. Yukari Hara and Ms. Chizuko Sasaki for skillful technical support.

Ebneter, A., Chidlow, G., Wood, J. P., and Casson, R. J. (2011). Protection of retinal ganglion cells and the optic nerve during short-term hyperglycemia in experimental glaucoma. Arch. Ophthalmol. 129, 1337-1344. doi: 10. 1001/archophthalmol.2011.269

Francis, B. A., Varma, R., Chopra, V., Lai, M. Y., Shtir, C., and Azen, S. P. (2008). Intraocular pressure, central corneal thickness and prevalence of open-angle glaucoma: the los angeles latino eye study. Am. J. Ophthalmol. 146, 741-746. doi: 10.1016/j.ajo.2008.05.048

Gordon, M. O., Beiser, J. A., Brandt, J. D., Heuer, D. K., Higginbotham, E. J., Johnson, C. A., et al. (2002). The ocular hypertension treatment study: baseline factors that predict the onset of primary open-angle glaucoma. Arch. Ophthalmol. 120, 714-720. doi: 10.1001/archopht.120.6.714

Han, G., Wood, J. P., Chidlow, G., Mammone, T., and Casson, R. J. (2013). Mechanisms of neuroprotection by glucose in rat retinal cell cultures subjected to respiratory inhibition. Invest. Ophthalmol. Vis. Sci. 54, 7567-7577. doi: 10. 1167/iovs.13-12200

Kabeya, Y., Mizushima, N., Ueno, T., Yamamoto, A., Kirisako, T., Noda, T., et al. (2000). LC3, a mammalian homologue of yeast Apg8p, is localized in autophagosome membranes after processing. EMBO J. 19, 5720-5728. doi: 10. 1093/emboj/19.21.5720

Kim, S. H., Munemasa, Y., Kwong, J. M., Ahn, J. H., Mareninov, S., Gordon, L. K., et al. (2008). Activation of autophagy in retinal ganglion cells. J. Neurosci. Res. 86, 2943-2951. doi: 10.1002/jnr.21738

Kitaoka, Y., Hayashi, Y., Kumai, T., Takeda, T., Munemasa, Y., Fujino, H., et al. (2009). Axonal and cell body protection by nicotinamide adenine dinucleotide in tumor necrosis factor-induced optic neuropathy. J. Neuropathol. Exp. Neurol. 68, 915-927. doi: 10.1097/NEN.0b013e3181afecfa

Kitaoka, Y., Kitaoka, Y., Kwong, J. M., Ross-Cisneros, F. N., Wang, J., Tsai, R. K., et al. (2006). TNF-alpha-induced optic nerve degeneration and nuclear factor-kappaB p65. Invest. Ophthalmol. Vis. Sci. 47, 1448-1457. doi: 10 1167/iovs.05-0299 
Kitaoka, Y., Munemasa, Y., Hayashi, Y., Kuribayashi, J., Koseki, N., Kojima, K., et al. (2011). Axonal protection by $17 \beta$-estradiol through thioredoxin1 in tumor necrosis factor-induced optic neuropathy. Endocrinology 152, 2775-2785. doi: 10.1210/en.2011-0046

Kitaoka, Y., Munemasa, Y., Kojima, K., Hirano, A., Ueno, S., and Takagi, H. (2013). Axonal protection by Nmnat3 overexpression with involvement of autophagy in optic nerve degeneration. Cell Death Dis. 4:e860. doi: 10. 1038/cddis.2013.391

Klein, B. E., Klein, R., and Jensen, S. C. (1994). Open-angle glaucoma and olderonset diabetes. The beaver dam eye study. Ophthalmology 101, 1173-1177. doi: 10.1016/s0161-6420(94)31191-2

Knöferle, J., Koch, J. C., Ostendorf, T., Michel, U., Planchamp, V., Vutova, P., et al. (2010). Mechanisms of acute axonal degeneration in the optic nerve in vivo. Proc. Natl. Acad. Sci. U S A 107, 6064-6069. doi: 10.1073/pnas.0909794107

Kojima, K., Kitaoka, Y., Munemasa, Y., Hirano, A., Sase, K., and Takagi, H. (2014). Axonal protection by modulation of p62 expression in TNF-induced optic nerve degeneration. Neurosci. Lett. 581, 37-41. doi: 10.1016/j.neulet.2014.08. 021

Kojima, K., Kitaoka, Y., Munemasa, Y., and Ueno, S. (2012). Axonal protection via modulation of the amyloidogenic pathway in tumor necrosis factorinduced optic neuropathy. Invest. Ophthalmol. Vis. Sci. 53, 7675-7683. doi: 10. 1167/iovs.12-10271

Kruse, R., Vind, B. F., Petersson, S. J., Kristensen, J. M., and Højlund, K. (2015). Markers of autophagy are adapted to hyperglycaemia in skeletal muscle in type 2 diabetes. Diabetologia 58, 2087-2095. doi: 10.1007/s00125-015-3654-0

Leske, M. C., Connell, A. M., Schachat, A. P., and Hyman, L. (1994). The barbados eye study. Prevalence of open angle glaucoma. Arch. Ophthalmol. 112, 821-829. doi: 10.1001/archopht.1994.01090180121046

Leske, M. C., Connell, A. M., Wu, S. Y., Hyman, L. G., and Schachat, A. P. (1995). Risk factors for open-angle glaucoma. The barbados eye study. Arch. Ophthalmol. 113, 918-924. doi: 10.1001/archopht.1995.011000700 92031

Leske, M. C., Wu, S. Y., Hennis, A., Honkanen, R., and Nemesure, B. (2008). Risk factors for incident open-angle glaucoma: the barbados eye studies. Ophthalmology 115, 85-93. doi: 10.1016/j.ophtha.2007. 03.017

Lv, P., Huang, J., Yang, J., Deng, Y., Xu, J., Zhang, X., et al. (2014). Autophagy in muscle of glucose-infusion hyperglycemia rats and streptozotocin-induced hyperglycemia rats via selective activation of $\mathrm{m}$-TOR or FoxO3. PLoS One 9:e87254. doi: 10.1371/journal.pone.0087254

Maday, S., Wallace, K. E., and Holzbaur, E. L. (2012). Autophagosomes initiate distally and mature during transport toward the cell soma in primary neurons. J. Cell Biol. 196, 407-417. doi: 10.1083/jcb.201106120

Margulis, J., and Finkbeiner, S. (2014). Proteostasis in striatal cells and selective neurodegeneration in huntington's disease. Front. Cell. Neurosci. 8:218. doi: 10. 3389/fncel.2014.00218

Menzies, F. M., Fleming, A., and Rubinsztein, D. C. (2015). Compromised autophagy and neurodegenerative diseases. Nat. Rev. Neurosci. 16, 345-357. doi: 10.1038/nrn3961

Mitchell, P., Smith, W., Chey, T., and Healey, P. R. (1997). Open-angle glaucoma and diabetes: the blue mountains eye study, australia. Ophthalmology 104, 712-718. doi: 10.1016/S0161-6420(97)30247-4

Mizushima, N., Levine, B., Cuervo, A. M., and Klionsky, D. J. (2008). Autophagy fights disease through cellular self-digestion. Nature 451, 1069-1075. doi: 10. 1038/nature06639

Mizushima, N., Yoshimori, T., and Levine, B. (2010). Methods in mammalian autophagy research. Cell 140, 313-326. doi: 10.1016/j.cell.2010.01.028

Munemasa, Y., and Kitaoka, Y. (2013). Molecular mechanisms of retinal ganglion cell degeneration in glaucoma and future prospects for cell body and axonal protection. Front. Cell. Neurosci. 6:60. doi: 10.3389/fncel.2012. 00060

Munemasa, Y., and Kitaoka, Y. (2015). Autophagy in axonal degeneration in glaucomatous optic neuropathy. Prog. Retin. Eye Res. 47, 1-18. doi: 10.1016/j. preteyeres.2015.03.002

Nakazawa, T., Nakazawa, C., Matsubara, A., Noda, K., Hisatomi, T., She, H., et al. (2006). Tumor necrosis factor- $\alpha$ mediates oligodendrocyte death and delayed retinal ganglion cell loss in a mouse model of glaucoma. J. Neurosci. 26, 12633-12641. doi: 10.1523/jneurosci.2801-06.2006
Park, H. Y., Kim, J. H., and Park, C. K. (2012). Activation of autophagy induces retinal ganglion cell death in a chronic hypertensive glaucoma model. Cell Death Dis. 3:e290. doi: 10.1038/cddis.2012.26

Rodríguez-Muela, N., Germain, F., Mariño, G., Fitze, P. S., and Boya, P. (2012). Autophagy promotes survival of retinal ganglion cells after optic nerve axotomy in mice. Cell Death Differ. 19, 162-169. doi: 10.1038/cdd.2011.88

Sawada, H., Fukuchi, T., Tanaka, T., and Abe, H. (2010). Tumor necrosis factor$\alpha$ concentrations in the aqueous humor of patients with glaucoma. Invest. Ophthalmol. Vis. Sci. 51, 903-906. doi: 10.1167/iovs.09-4247

Sommer, A., and Tielsch, J. M. (1996). Risk factors for open-angle glaucoma: the barbados eye study. Arch. Ophthalmol. 114:235. doi: 10.1001/archopht.1996. 01100130229029

Tezel, G., Li, L. Y., Patil, R. V., and Wax, M. B. (2001). TNF-alpha and TNF-alpha receptor-1 in the retina of normal and glaucomatous eyes. Invest. Ophthalmol. Vis. Sci. 42, 1787-1794.

Tezel, G., and Wax, M. B. (2000). Increased production of tumor necrosis factor- $\alpha$ by glial cells exposed to simulated ischemia or elevated hydrostatic pressure induced apoptosis in cocultured retinal ganglion cells. J. Neurosci. 20, 8693-8700.

Tielsch, J. M., Katz, J., Quigley, H. A., Javitt, J. C., and Sommer, A. (1995). Diabetes, intraocular pressure and primary open-angle glaucoma in the baltimore eye survey. Ophthalmology 102, 48-53. doi: 10.1016/s0161-6420(95)31055-x

Topouzis, F., Wilson, M. R., Harris, A., Founti, P., Yu, F., Anastasopoulos, E., et al. (2011). Risk factors for primary open-angle glaucoma and pseudoexfoliative glaucoma in the thessaloniki eye study. Am. J. Ophthalmol. 152, 219.e1-228.e1. doi: 10.1016/j.ajo.2011.01.032

Wei, N., Yu, S. P., Gu, X. H., Chen, D. D., Whalin, M. K., Xu, G. L., et al. (2013). The involvement of autophagy pathway in exaggerated ischemic brain damage in diabetic mice. CNS Neurosci. Ther. 19, 753-763. doi: 10.1111/cns.12123

$\mathrm{Wu}, \mathrm{K} . \mathrm{K}$., and Huan, Y. (2008). Streptozotocin-induced diabetic models in mice and rats. Curr. Protoc. Pharmacol. Chapter 5, Unit 5.47. doi: 10 1002/0471141755.ph0547s70

Yan, X., Tezel, G., Wax, M. B., and Edward, D. P. (2000). Matrix metalloproteinases and tumor necrosis factor alpha in glaucomatous optic nerve head. Arch. Ophthalmol. 118, 666-673. doi: 10.1001/archopht.118.5.666

Yang, S., Xia, C., Li, S., Du, L., Zhang, L., and Hu, Y. (2014). Mitochondrial dysfunction driven by the LRRK2-mediated pathway is associated with loss of Purkinje cells and motor coordination deficits in diabetic rat model. Cell Death Dis. 5:e1217. doi: 10.1038/cddis.2014.184

Yu, N., Maciejewski-Lenoir, D., Bloom, F. E., and Magistretti, P. J. (1995). Tumor necrosis factor-alpha and interleukin-1 alpha enhance glucose utilization by astrocytes: involvement of phospholipase A2. Mol. Pharmacol. 48, 550-558.

Yuan, L., and Neufeld, A. H. (2000). Tumor necrosis factor-alpha: a potentially neurodestructive cytokine produced by glia in the human glaucomatous optic nerve head. Glia 32, 42-50. doi: 10.1002/1098-1136(200010)32:1<42::aidglia40>3.0.co;2-3

Zhang, T., Liu, X., Li, Q., Wang, J., Jia, W., and Sun, X. (2010). Exacerbation of ischemia-induced amyloid-beta generation by diabetes is associated with autophagy activation in mice brain. Neurosci. Lett. 479, 215-220. doi: 10.1016/j. neulet.2010.05.064

Zhao, D., Cho, J., Kim, M. H., Friedman, D. S., and Guallar, E. (2015). Diabetes, fasting glucose and the risk of glaucoma: a meta-analysis. Ophthalmology 122 , 72-78. doi: 10.1016/j.ophtha.2014.07.051

Zhou, M., Wang, W., Huang, W., and Zhang, X. (2014). Diabetes mellitus as a risk factor for open-angle glaucoma: a systematic review and meta-analysis. PLoS One 9:e102972. doi: 10.1371/journal.pone.0102972

Conflict of Interest Statement: The authors declare that the research was conducted in the absence of any commercial or financial relationships that could be construed as a potential conflict of interest.

Copyright (c) 2015 Sase, Kitaoka, Munemasa, Kojima and Takagi. This is an openaccess article distributed under the terms of the Creative Commons Attribution License (CC BY). The use, distribution and reproduction in other forums is permitted, provided the original author(s) or licensor are credited and that the original publication in this journal is cited, in accordance with accepted academic practice. No use, distribution or reproduction is permitted which does not comply with these terms. 